\title{
LARGE MAGELLANIC CLOUD CARBON STARS: READING THE ROSETTA STONE OF STELLAR EVOLUTION
}

\author{
JAY A. FROGEL \\ Department of Astronomy, The Ohio State University \\ Columbus, $\mathrm{OH}$ 43210, U.S.A.; and \\ Physics Department, University of Durham \\ Durham, England \\ AND \\ EDGARDO COSTA \\ Departamento de Astronomia, Universidad de Chile \\ Santiago, Chile
}

\begin{abstract}
We discuss new results based on $R I$ and $J H K$ photometry for 888 and 204 carbon stars, respectively, of the $1035 \mathrm{C}$ stars found by Blanco and his collaborators in the Large Magellanic Cloud (LMC). Bolometric magnitudes and effective temperatures for these stars are calculated and compared with theoretical predictions. We find a spatial gradient in the transition luminosity between $\mathrm{M}$ and $\mathrm{C}$ type stars. This has implications for the age of the most recent major epoch of star formation in the LMC.
\end{abstract}

\section{Introduction and Rationale}

Just as the Rosetta Stone provided the key to understanding a previously incomprehensible symbolic language (Uhlemann 1853), so too the Magellanic Clouds have been the key to the interpretation of the visible signs of stellar evolution. This has been particularly true for the final stages of a star's existence. A spectacular recent example has of course been SN 1987A. Much less glamorous but perhaps of equal importance has been the extensive study of asymptotic giant branch (AGB) stars in the Clouds with a continual interplay between theory and observation. In this review we will concentrate on the critical role the Magellanic Clouds have played in 
the development of a coherent picture for the formation and evolution of carbon (C) stars on the AGB.

First, consider several reasons why understanding the evolution of $\mathrm{C}$ stars should be of interest to other than the specialist: $\mathrm{C}$ stars are the final stage of AGB evolution for most if not all stars with masses between 1 and $7 M_{\odot}$. They are the major source of carbon-rich molecules and grains for the interstellar medium. Carbon stars dominate the bolometric luminosity and the near-IR light of intermediate-age stellar systems which, in turn, implies that they can be an excellent age diagnostic for integrated-light observations of stellar clusters and galaxies.

The importance of the Magellanic Clouds in C-star research comes about because we can get complete samples of AGB C stars in the Clouds, i.e. samples which are not magnitude-limited (e.g. Blanco et al. 1980, hereafter BMB; Blanco \& McCarthy 1983, hereafter BM; Rebeirot et al. 1993). The distance to the Clouds is known to a relatively high degree of accuracy and their distension along the line of sight is small relative to their distance. These facts combined with a low and relatively uniform reddening to each Cloud imply that accurate absolute luminosity functions can be derived for $\mathrm{C}$ stars. Finally, the LMC and SMC have significant numbers of populous star clusters which contain large numbers of AGB stars (of both $\mathrm{C}$ and $\mathrm{M}$ type) and whose ages and chemical compositions can be well estimated and are closely related to one another (e.g. Cohen 1982; Bica et al. 1986).

Blanco and his collaborators (BM \& BMB) identified more than 1000 $\mathrm{C}$ stars in the course of their low dispersion red spectroscopic surveys for $\mathrm{C}$ and M giants in 52 fields in the LMC. We (Costa \& Frogel 1996) have obtained $R I$ photometry for an unbiased sample of about 900 of these $\mathrm{C}$ stars and $J H K$ photometry for about 200 of the stars with $R I$ data. Our objectives in undertaking such an extensive program of single-channel photometry fall into two general categories: first, to empirically derive physical properties of LMC C stars with a sample that is nearly an order of magnitude larger than any previous one and to compare these properties with predictions of stellar evolution theory; and second, to see what can be learned about the history of star formation in the LMC by searching for spatial dependences of these properties.

\section{The Luminosity Function for LMC Field C Stars}

We (Costa \& Frogel 1996) have calculated accurate bolometric magnitudes for the $197 \mathrm{C}$ stars observed at RIJHK. With these calculations and the tight relation between $\mathrm{BC}_{K}$ and $J-K$ that exists for both $\mathrm{M}$ and $\mathrm{C}$ stars (Frogel et al. 1980), it is possible to derive an equation which relates the difference between the $I$ magnitude and $m_{\text {bol }}$ to the $R-I$ color with an 


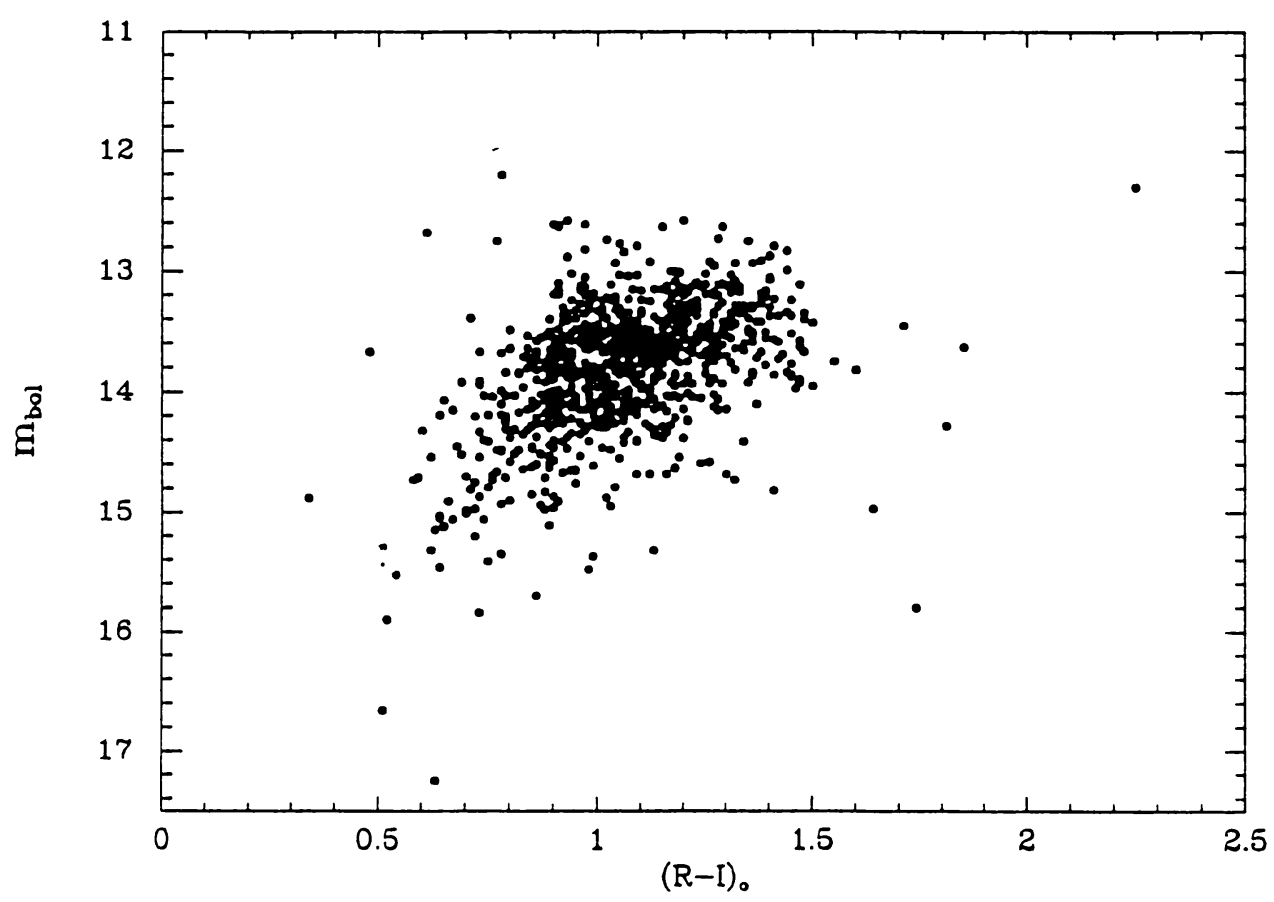

Figure 1. The $m_{\text {bol }}$ vs. ( $R-I$ )。 color-magnitude diagram (Costa \& Frogel 1996) for 888 $\mathrm{C}$ stars from the surveys of BMB and BM. The upper and lower magnitude limits to the distribution of stars is intrinsic and not due to observational selection effects.

uncertainty of $\pm 0.34 \mathrm{mag}$. Figure 1 is a color-magnitude diagram for the unbiased sample of $888 \mathrm{C}$ stars from the Blanco et al. surveys for which we have obtained $R I$ photometry and calculated bolometric magnitudes. Figure 2 is the luminosity function for these stars.

The cluster $\mathrm{C}$ star luminosity function, as well as the field $\mathrm{C}$ star function of Cohen et al. (1981, hereafter CFPE), both of which were based on samples of fewer than 100 stars, are statistically indistinguishable from the new LMC field C star function based on nearly 1000 stars. Thus, the comparisons of the first two functions with stellar models by, inter alia, Iben (1981), Iben \& Renzini (1983), Lattanzio (1986), and Groenewegen \& de Jong (1993) remain valid.

Our new results (Costa \& Frogel 1996) emphasize the nearly complete absence of $C$ stars with $m_{\text {bol }} \leq 12.5$ - there are only 2 in our entire sample, or $\sim 0.2 \%$. Since the Blanco et al. surveys may have missed a small number of $\mathrm{C}$ stars at the two extremes of the color distribution, the actual number of luminous stars may be slightly greater than $0.2 \%$. This limit to the presence of any significant population of luminous $\mathrm{C}$ stars in the field 


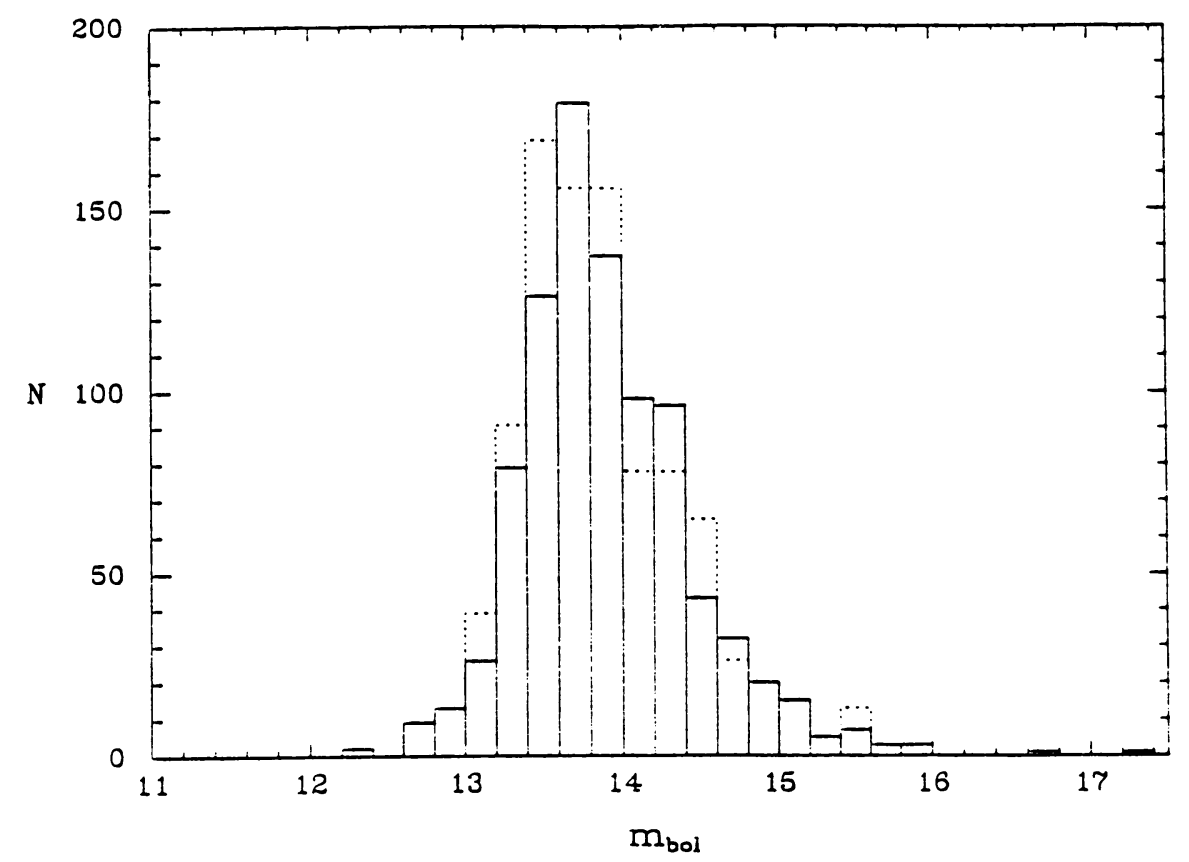

Figure 2. The apparent bolometric luminosity function for the 395 L.MC field C stars from Figure 1 is shown by the solid line. The dashed line is the same function for the 69 C stars identified in SWB 3.5-6.5 clusters of the Magellanic Clouds by FMB. This later function has been scaled by $895 / 69$ to facilitate comparison.

of the LMC, when combined with results from Frogel \& Blanco (1990) on field M stars and from Frogel et al. (1990, hereafter FMB) for clusters, leads us to conclude that one or more processes such as a superwind phase or convective overshooting strongly inhibits AGB stars in the LMC from becoming brighter than $M_{\text {bol }} \approx-6$. There is no evidence that the missing bright $\mathrm{C}$ stars have reverted back to $\mathrm{M}$ stars. Similar results come from the surveys of Reid et al. (1990) for luminous AGB stars in the LMC, of Reid \& Mould (1990) in the SMC, and of Wood et al. (1992) for OH/IR stars.

Since the Blanco et al. surveys are not magnitude-limited, we can also draw rather definitive conclusions regarding the faint end of the luminosity function. From our sample of $\sim 900$ field C stars, only $3.8 \%$ are fainter than $m_{\text {bol }}=15$. Examination of the color-magnitude diagram in Figure 1 shows that most of these bolometrically faint stars are also quite blue and will tend to be among the faintest stars in $I$ as well. Thus. since effects due to misidentification (some could be $\mathrm{M}$ stars) and crowding could become important for these stars, 15.0 or 15.2 are good estimates for the faint limit to the $\mathrm{C}$ star luminosity function in the LMC. 


\section{Colors and Effective Temperatures of LMC Field C Stars}

We have just shown that the field and cluster $\mathrm{C}$ stars have indistinguishable distributions in bolometric magnitude. Now consider their colors. Figure 3 compares the $J-H, H-K$ colors of the field stars from Costa \& Frogel with the colors of cluster stars from FMB. The extent of the cluster star distribution in Figure 3 is well covered by that of the field $\mathrm{C}$ stars. But although they have similar blue limits to their distributions, the field stars extend to considerably redder colors than do the clusters stars: there are only two cluster C stars, both of the LMC, redward of $H-K=0.75$. This difference is not simply due to a difference in sample size. Fully $11 \%$ of the field C star sample have $J-K \geq 1.9$ while only $3 \%$ of the cluster sample is this red. FMB have also noted that the LMC Bar West field has a higher percentage of red, luminous $M$ stars than their cluster $M$ star sample.

An excess of red $\mathrm{M}$ and $\mathrm{C}$ stars in the field could arise from a metallicity distribution skewed to higher values in the field than in the cluster sample. Higher metallicity produces redder $\mathrm{C}$ stars since the evolutionary track of such a star will be shifted to cooler temperatures than that of a lower metallicity star of the same luminosity (as will the tracks of $M$ stars). Also, the formation of molecules and grains will be enhanced in the higher metallicity star due to both lower temperatures and larger numbers of heavy atoms. These enhancements could, in turn, result in increased blanketing by molecular absorption bands, higher mass-loss rates (cf. Groenewegen et al. 1995), and increased circumstellar thermal emission. For the same age, a higher metallicity star will have a greater mass than a lower metallicity star; thus, its AGB evolution would terminate at a brighter magnitude. Also, the luminosity which marks the transition between $\mathrm{M}$ and $\mathrm{C}$ stars would be brighter in the more metal-rich population, thus accounting for the elevated luminosity of the red field $M$ stars.

Except for their bluer colors, the luminous $\mathrm{CH}$ stars found by Hartwick \& Cowley (1988) in the LMC (for which infrared data are given by Suntzeff et al. 1993 and Feast \& Whitelock 1992) appear to be indistinguishable in terms of their measureable properties from the redder $\mathrm{C}$ stars studied by Costa \& Frogel (1996). They constitute only a small percentage of the total $\mathrm{C}$ star population of the LMC and we propose that these $\mathrm{CH}$ stars are just the blue tail of the distribution of redder $\mathrm{C}$ stars rather than a distinct class of stars.

Costa \& Frogel (1996) derived effective temperatures for the C stars from their $R-I$ and $J-K$ colors. Three different estimates yield results with a mean dispersion less than $150 \mathrm{~K}$ for an individual star. A comparison of the absolute magnitude, $T_{\text {eff }}$ diagram with theoretical tracks indicates that a $1 M_{\odot}$ model does indeed represent the lower mass limit for a moderately 


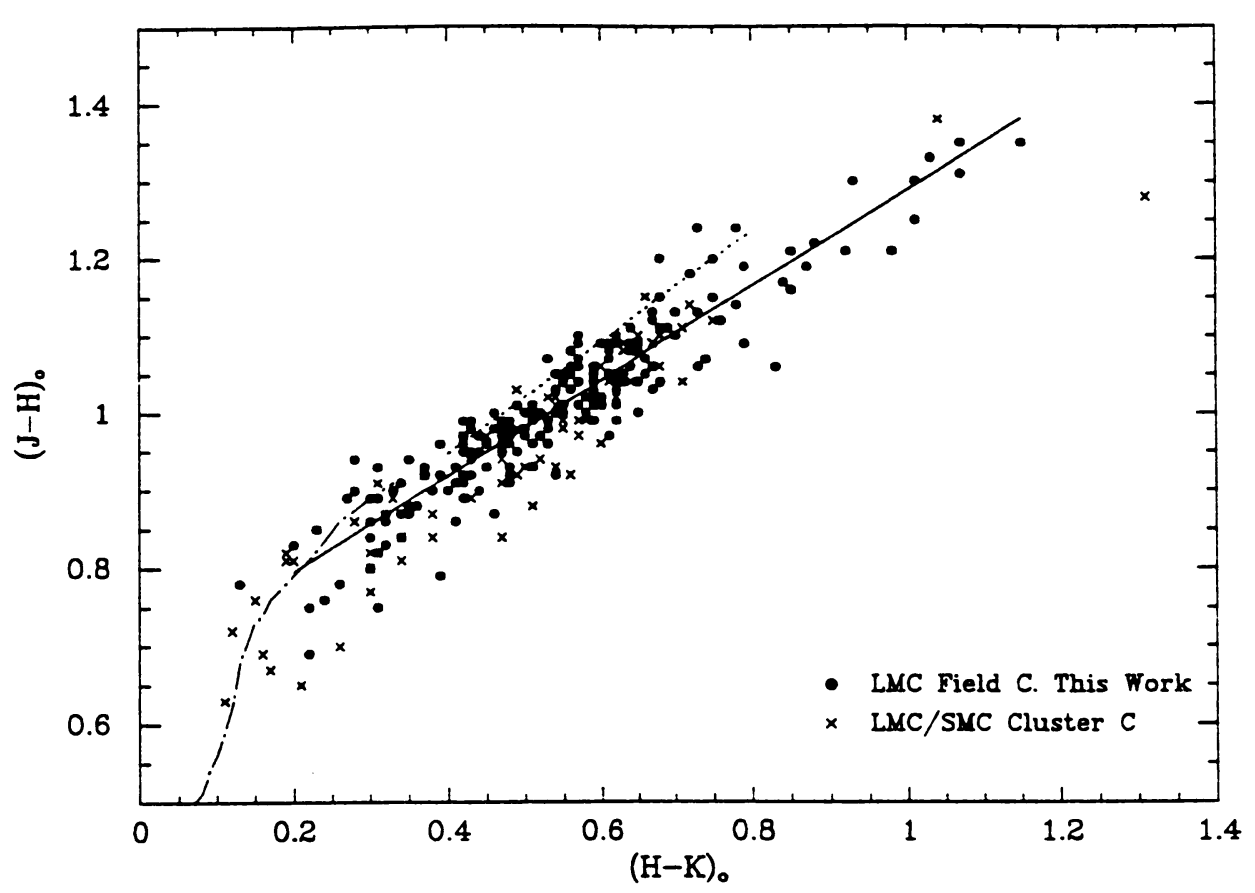

Figure 3. The near-IR color-color diagram for the $204 \mathrm{LMC}$ field C stars with JHK photometry from Costa \& Frogel (1996, filled circles). C stars in clusters of the Magellanic Clouds (FMB) are also plotted. The least-squares fit to the field data is the solid line. The mean relation for galactic $C$ stars is the dotted straight line (CFPE); for galactic $M$ stars it is the dot-dashed curved line (Frogel et al. 1978).

metal-poor C star (cf. Lattanzio 1989; FMB; see also Fig. 4 in Westerlund et al. 1995). In lower mass stars, dredge-up of processed material is not sufficient to bring about $\mathrm{C} / \mathrm{O}>1$ in the envelope. To turn a more metal-rich or younger (i.e. more massive) $\mathrm{M}$ star into a $\mathrm{C}$ star requires a combination of higher luminosity and lower temperature.

\section{The Transition Luminosity between M and C Stars: Implications for the Star Formation History of the LMC}

FMB show that for Magellanic Cloud clusters that contain both $\mathrm{C}$ and $M$ stars, there exists a transition luminosity $m_{\text {bol }}(t)$ between the faintest $C$ stars and the brightest $M$ stars, and that this transition luminosity is correlated with the cluster's SWB type (Searle et al. 1980) and, hence, with its age and metallicity in the sense that the transition luminosity increases with increasing $Z$ and decreasing age. Costa \& Frogel estimated $m_{\text {bol }}(\mathrm{t})$ values for each field in which at least $8 \mathrm{C}$ stars were observed 
by averaging the magnitudes of the 4 faintest $\mathrm{C}$ stars. These estimates are almost all between $m_{\mathrm{bol}}(\mathrm{t})=14.0$ and 15.0, comparable to the fainter transition luminosities for SWB V-VI type Cloud clusters (see FMB's Table 3 and Fig. 14) and significantly fainter than the transition luminosities of the earlier, III-IV, SWB types which are between 13.1 and 13.5. Since the technique used by FMB to calculate transition luminosities involved averaging the $m_{\mathrm{bol}}$ of the faintest $\mathrm{C}$ stars with the $m_{\mathrm{bol}}$ of the brightest $M$ stars, and since the brightest $M$ stars are typically fainter than the faintest $\mathrm{C}$ stars in any given cluster by a few tenths of a magnitude, the $m_{\text {bol }}(\mathrm{t})$ values calculated by Costa \& Frogel (1996) would need to be made yet fainter for a proper comparison with the FMB values. Given the close linkage between age and metallicity for the clusters (e.g. Cohen 1982; Bica et al. 1986) this result implies that throughout the LMC there is a significant population of stars similar to those found in the older, metal-poor end of the distribution of intermediate age LMC clusters. In other words, none of the fields surveyed by BM has an exclusively younger stellar population such as is found in clusters of SWB types II-IV.

Figure 4 shows a significant brightening of $m_{\text {bol }}(\mathrm{t})$ with increasing distance from the center of the LMC. The change in $m_{\text {bol }}(t)$ is from $\sim 14.7$ near the Bar to $\sim 14.1$ in the outermost fields. If we compare this with the relation between $m_{\text {bol }}(t)$ and turnoff mass in Fig. 19 of FMB based on clusters of known ages and metallicities, we can estimate the oldest epoch of $\mathrm{C}$ star formation as a function of position. To effect this comparision the present values of $m_{\text {bol }}(t)$ must be made fainter by a few tenths of a magnitude to take into account the fact that our estimates for $m_{\text {bol }}(t)$ were made without the help of any $\mathrm{M}$ stars. We conclude that if the field $\mathrm{C}$ stars follow the same $[\mathrm{Fe} / \mathrm{H}]$ - age relation as the clusters, then the earliest epoch of C-star formation near the Bar of the LMC corresponds to clusters of SWB type 6.5 , while near the periphery it corresponds to clusters of type 5.5, or an age difference of a few Gyr according to Table 3 of FMB (a younger "earliest epoch" on the periphery than near the Bar) and an $[\mathrm{Fe} / \mathrm{H}]$ difference of 0.2 to $0.4 \mathrm{dex}$ (a higher $[\mathrm{Fe} / \mathrm{H}]$ near the periphery). If the mean $[\mathrm{Fe} / \mathrm{H}]$ at the periphery were forced to be the same as that near the Bar, the implied age difference would be greater, i.e. the periphery would be younger still. For reasons we do not fully understand, the dispersion in the relationship between $m_{\mathrm{bol}}(\mathrm{t})$ and distance is considerably less if fields containing populous clusters are excluded from the analysis. This may have to do with $\mathrm{C}$ stars from the clusters contaminating the field $\mathrm{C}$ stars.

Carbon stars are just the tip of the iceberg that represents a major epoch of star formation occurring a few Gyr ago in the LMC and first identified by Butcher (1977). Our finding of a systematic spatial variation in $m_{\text {bol }}(\mathrm{t})$ suggests that this epoch occurred more recently in the periphery 


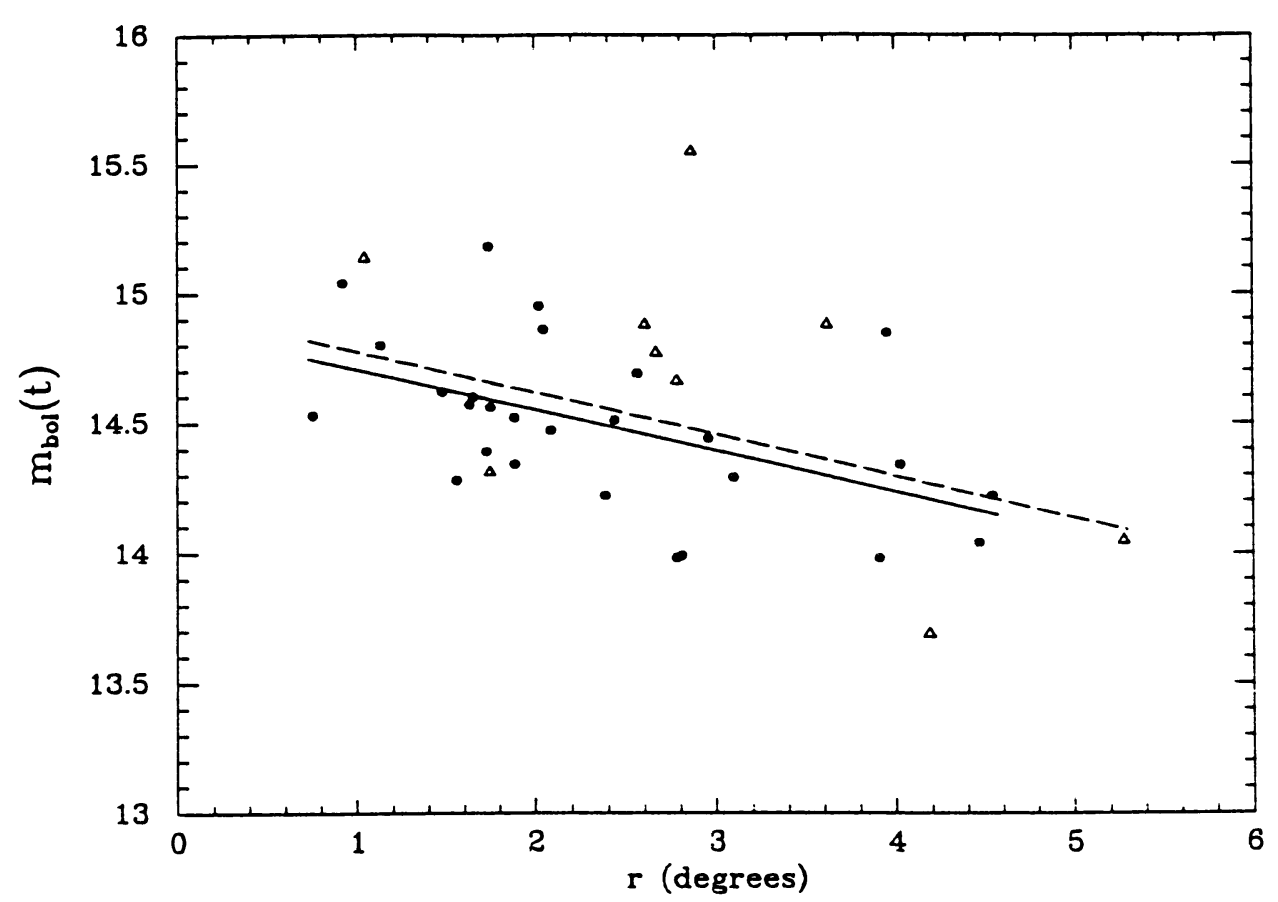

Figure 4. The transition luminosity $m_{\text {bol }}(t)$ for the 36 fields which have 8 or more $C$ stars observed by us, shown as a function of the field's radial distance from the center of the LMC. Solid dots represent fields without clusters; open triangles fields centered on populous clusters. The dashed line is the linear regression of $m_{\text {bol }}(t)$ on $r$ for all 36 fields. The solid line is the same regression for only the 27 fields without clusters.

than in the central regions of the LMC. This result could be tested by obtaining optical CMDs that include the main sequence turnoff. Given the variation in $m_{\text {bol }}(t)$, one would expect to find a variation in the $C$ to $M$ star ratio as well. However, in view of the small size of the change in $m_{\text {bol }}(t)$, examination of the cluster data in FMB indicates that the absence of a variation in the $C$ to $M$ star ratio $(B M)$ is not unexpected and can be attributed to statistical fluctuations in star numbers. BM did find large spatial gradients in the $C$ to $M$ star ratio in the SMC. Thus it would be useful to carry out a study similar to ours of the $\mathrm{C}$ stars they identified in the SMC.

JAF was a staff member and EC a visiting astronomer at CTIO when these observations were made. CTIO is operated by AURA, Inc. under contract with the NSF. JAF also thanks Roger Davies and PPARC for a Visiting Senior Research Fellowship at the Univeristy of Durham where this review was prepared. 


\section{References}

Bica, E., Dottori, H. \& Pastoriza, M. 1986, $A \& A, 156,261$

Blanco, V.M. \& McCarthy, M.F. 1983, $A J, 88,1442$ (BM)

Blanco, V.M., McCarthy, M.F. \& Blanco, B. M. 1980, ApJ, 242, 938 (BMB)

Butcher, H. 1977, ApJ, 216, 372

Cohen, J.G. 1982, ApJ, 258, 143

Cohen, J. G., Frogel, J. A., Persson, S. E. \& Elias, J.H. 1981, ApJ, 249, 481 (CFPE)

Costa, E. \& Frogel, J. A. 1996, $A J ; 112,2607$

Feast, M. W. \& Whitelock, P. A. 1992, MNRAS, 259, 6

Frogel, J. A. \& Blanco, V. M. 1990, ApJ, 365, 168

Frogel, J. A., Mould, J. \& Blanco, V.M. 1990, ApJ, 352, 96 (FMB)

Frogel, J. A., Persson, S.E., Aaronson, M. \& Matthews, K. 1978, ApJ, 220, 75

Frogel, J. A., Persson, S. E. \& Cohen, J. G. 1980, $A p J, 239,495$

Groenewegen, M. A. T. \& de Jong, T. 1993, $A \& A, 267,410$

Groenewegen, M. A. T., van den Hoek, L. B. \& de Jong, T. 1995, $A \mathscr{E} A, 293,381$

Hartwick, F. D. A. \& Cowley, A. P. 1988, ApJ, 334, 135

Iben, I., Jr. 1981, $A p J, 246,278$

Iben, I., Jr. \& Renzini, A. 1983, Ann. Rev. Astron. Astrophys., 21, 271

Lattanzio, J. C. 1986, $A p J, 311,708$

Lattanzio, J. C. 1989, ApJ, 344, L25

Rebeirot, E., Azzopardi, M. \& Westerlund, B.E. 1993, A\&A Supp., 97, 603

Reid, N. \& Mould, J. 1990, ApJ, 360, 490

Reid, N., Tinney, C. \& Mould, J. 1990, ApJ, 348, 98

Searle, L., Wilkinson, A. \& Bagnuolo, W.G. 1980, ApJ, 239, 803 (SWB)

Suntzeff, N. B., Phillips, M. M., Elias, J. H., Cowley, A. P., Hartwick, F. D. A. \& Bouchet, P. 1993, PASP, 105, 350

Uhlemann, M. 1853, "Inscriptionis Rosettanae Hieroglyphicae Decretum Sacerdotale," (Lipsiae: Dykiana)

Westerlund, B.E., Azzopardi, M., Breysacher, J. \& Rebeirot, E. 1995, A\&A, 303, 107

Wood, P. R., Whiteoak, J.B., Hughes, S. M. G., Bessell, M.S., Gardner, F. F. \& Hyland, A. R. $1992, A p J, 397,552$

\section{Discussion}

Gustafsson: What does the tendency of $m_{\text {bol }}$ to vary with distance from the LMC Bar correspond to in terms of age differences?

Frogel: The difference is $\sim 30 \%$ in turnoff mass. If SWB III clusters have an age of a few hundred $\mathrm{Myr}$, then the age difference from Bar to edge is a couple of Gyr or $30-50 \%$.

Gustafsson: Is it possible to separate age effects from metallicity effects for the LMC clusters and from that deduce the significance of metallicity alone for the $\mathrm{C}$ star phenomenon? I suppose that would be difficult?

Frogel: We have "to assume that the age $-[\mathrm{Fe} / \mathrm{H}]$ relation in clusters is one-to-one since the scatter is comparable to the uncertainties. This means we assume the same correlation for $\mathrm{C}$ stars. The dependence noted in $m_{\text {bol }}$ (transition) with distance may change this. 
Kerschbaum: With your near-IR sample, how can you be sure not to miss the high-mass-loss objects? Also, with only near-IR data the estimation of $m_{\text {bol }}$ should be more uncertain for higher-mass-loss objects. To avoid these problems, two ISO projects, namely by Trams and by Loup et al., are studying AGB stars in the LMC in the mid- and far-IR.

Frogel: An $L$-band survey that Harvey Richer and I did a long time ago did not reveal any significant number of "hidden" C stars. Also, as part of our cluster work we scanned many of the clusters in the near-IR on the CTIO 4-m and found only one star that is too faint in the $I$ band to have been detected by the Blanco et al. surveys. Third, Reid \& Mould concluded, on the basis of a deep $I$ band imaging survey, that the numbers of dust-enshrouded C stars in the LMC and SMC have to be very small. 